\title{
Gérer ou gouverner la ressource en eau ? Modélisation en temps réel et gestion de la pénurie au sein de la rivière Crocodile (Afrique du Sud)
}

\author{
Magalie Bourblanc ${ }^{1, a, *}$ et David Blanchon ${ }^{2}$ \\ 1 Politiste, Cirad, UMR G-EAU, Montpellier, France \\ 2 Géographe, Université Paris Ouest Nanterre, Faculté de Géographie, Nanterre, France
}

Reçu le $1^{\text {er }}$ mars 2016. Accepté le 6 avril 2017

Cet article s'intéresse à la pénurie en eau et aux instruments appliqués pour traiter de cet enjeu sur la rivière Crocodile en Afrique du Sud. Le texte s'inscrit dans la perspective d'une construction sociale de la pénurie, en mettant en évidence la manière dont différents dispositifs sociotechniques de gestion de la pénurie se transforment en instruments de gouvernement, suivant les objectifs politiques qui leurs sont assignés. Les auteurs montrent que loin de remédier aux problèmes pour lesquels ils ont été définis, certains de ces dispositifs pérennisent des inégalités dans l'accès aux ressources et renforcent le statu quo. Au-delà du contexte sud-africain, ce texte permet de souligner la prédominance d'une vision ingénériale dans le domaine de la gestion de l'eau, qui, malgré les tentatives pour mettre au point des modèles censés objectiver la gestion en temps réel et le calcul des débits environnementaux, n’en demeure pas moins tributaire de données lacunaires et de contraintes politiques fortes avec lesquelles elle doit composer.

La Rédaction

\begin{abstract}
Résumé - Nous analysons dans cet article trois dispositifs sociotechniques de gestion de la pénurie en eau mis en œuvre sur la rivière Crocodile en Afrique du Sud: un processus de réallocation de la ressource (compulsory licensing), un modèle informatisé de gestion en temps réel et une détermination plus précise du débit environnemental. Cette analyse est l'occasion de mettre en évidence à quel point ces dispositifs sociotechniques, loin de se contenter de gérer, d'un point de vue purement technique, la ressource en eau, se sont transformés en instruments de gouvernement de celle-ci, neutralisant certaines dispositions réglementaires et certains objectifs de politiques publiques au cœur de l'agenda de transformation du pays. En outre, nous démontrons que cette pénurie est tout autant sinon plus un construit, découlant des choix de gestion, qu'une réelle fatalité sur la Crocodile.
\end{abstract}

Mots-clés : environnement / gouvernance / dispositifs sociotechniques / pénurie en eau / Afrique du Sud

\begin{abstract}
Managing or governing water resources? Real-time model and the management of the scarcity on the Crocodile River (South Africa). In this article we analyse three sociotechnical devices that have been implemented to deal with water scarcity on the Crocodile River (South Africa): a resource re-allocation and redistribution process called "compulsory licensing", a computerised real-time management model and the operationalisation of environmental flows on a monthly and even daily basis. Our analysis highlights the extent to which such sociotechnical devices, far from managing water resources from a purely technical perspective, actually perform as instruments governing water resources. In that respect, they have been able to neutralise specific regulatory provisions and public policy objectives that were at the heart of South Africa's transformation agenda. In addition, we demonstrate that water scarcity does not represent a real fatality on the Crocodile River but largely stems from management decisions.
\end{abstract}

Keywords: environment / governance / sociotechnical devices / water scarcity / South Africa

\footnotetext{
${ }^{a}$ Magalie Bourblanc est également rattachée au Centre for the Study of Governance Innovation (GovInn), University of Pretoria, Pretoria, Afrique du Sud.

* Auteur correspondant : magalie.bourblanc@cirad.fr
} 
En 1995, dans leur chapitre d'ouvrage justement intitulé «Rivers and Streams in Southern Africa: predictably unpredictable », B. R. Davies, J. H. O'Keefe et C. Snaddon soulignaient que les cours d'eau étaient en Afrique du Sud parmi les plus marqués par la variabilité au monde, et que leur gestion posait donc des problèmes inconnus par rapport aux hydrosystèmes des pays plus tempérés. Ils affirmaient également que cette variabilité hydrologique était nécessaire au fonctionnement des écosystèmes, qui avaient besoin de ces crues et de ces étiages désormais effacés par le grand système de barrage et de transferts d'eau construit dans ce pays. "La variabilité et l'imprévisibilité, écrivent-ils, sont donc les caractéristiques les plus remarquables des rivières de la région [...], et la régulation phénoménale dont elles ont été l'objet est la principale menace qui pèse sur elles, en imposant une prévisibilité sur des systèmes imprévisibles » (Davies et al., 1995, p. 592).

À ce régime naturellement variable des cours d'eau sud-africains, s'ajoute depuis plus de vingt ans une profonde réorganisation de leur mode de gestion. Dès 1996 dans le livre blanc sur la gestion de l'eau, le ministre K. Asmal promouvait une gestion fondée sur un nouveau slogan «Some, For All, For Ever»- « un peu d'eau pour tous, pour toujours »- censé remplacer la gestion de l'eau du régime d'apartheid réservant toute l'eau à certains (les fermiers blancs notamment) selon une approche peu durable. Mais cette révolution, marquée par l'adoption d'une loi sur l'eau en 1998, le National Water Act (NWA), est restée largement inachevée, et quelques-uns de ses principaux dispositifs (agences de bassin, nouveaux modes d'attribution des droits à l'eau, mise en place d'une «ecological reserve» que l'on peut traduire par «débit environnemental», nouveaux modèles informatisés de gestion) n'ont été encore à ce jour que très partiellement mis en place. Et ce alors que les discours sur la pénurie croissante des ressources se multiplient.

Le bassin de la Crocodile River ${ }^{1}$, situé à l'extrémité orientale de l'Afrique du Sud, non loin de la frontière du Swaziland, est un laboratoire de cette «révolution» politique, puisque que c'est l'un des rares bassins versants où les principaux dispositifs de la loi sur l'eau de 1998 ont été mis en place, avec notamment la création d'une agence de bassin, l'Inkomati-Usutu Catchment Management Agency (IUCMA), le lancement d'un modèle informatisé de gestion en temps réel des cours d'eau et enfin le calcul d'un débit environnemental. Une caractéristique de ce cours d'eau, sur laquelle nous

\footnotetext{
${ }^{1}$ Il s'agit de la Crocodile River (East). Cette précision est utilisée pour la distinguer de la Crocodile River (West) située non loin de Johannesburg. Mais tous les acteurs locaux utilisent simplement la dénomination «Crocodile River».
}

reviendrons dans les prochaines sections, est qu'il s'agit d'un des bassins versants où le risque de "pénurie potentielle», selon le ministère de l'Eau, est le plus fort. Enfin, son régime «naturel» montre une forte variabilité hydrologique typique des cours d'eau de la région, avec également une modification importante du débit naturel par les barrages et les prélèvements dans le cours d'eau, même si la Crocodile reste moins «régulée» que d'autres hydrosystèmes sud-africains. Étant donné la forte pression subie en termes d'usages sur ce bassin, on peut s'attendre à ce que l'expertise autour de la situation hydrique du bassin et les calculs auxquels cette évaluation donne lieu pour déterminer un éventuel état de pénurie fassent l'objet d'âpres négociations.

L'objectif de cet article est d'analyser la manière dont la forte variabilité de la ressource en eau et l'apparition potentielle de pénuries dans ce bassin versant sont gérées, en nous focalisant sur trois dispositifs sociotechniques: celui du compulsory licensing, puis celui du développement d'un modèle de gestion en temps réel de la ressource, enfin celui du «débit environnemental». Par dispositif sociotechnique, nous entendons « un ensemble d'instruments techniques [... fortement imbriqués] avec le monde social qui les a produits et sur lequel ils agissent» pour reprendre la définition qu'en donne le courant de la sociologie des sciences (Zittoun, 2013). Ce concept est très proche de celui d' «instrument technique d'action publique» (Lascoumes et Le Galès, 2004) promu par l'analyse sociologique de l'action publique. Décrits comme un «dispositif à la fois technique et social», «nourris d'une interprétation du social et de conceptions précises du mode de régulation envisagé », ces instruments d'action publique sont loin de se résumer à des discussions techniques axiologiquement neutres, mais sont au contraire façonnés par des représentations idéologiques qui ont parfois peu à voir avec les objectifs d'action publique visés (Lascoumes et Le Galès, 2004, p. 13). L'approche de Lascoumes et Le Galès souligne toute l'importance d'éclairer la dimension politique de ces dispositifs ou instruments.

Après une brève présentation du bassin et de ses enjeux, nous étudions les difficultés liées à la mise en place du processus de compulsory licensing, sur lesquelles les gestionnaires de l'IUCMA ont su habilement jouer pour tenter d'écarter définitivement cet instrument réglementaire ${ }^{2}$. Nous analysons ensuite les modalités du recours au modèle real-time en soulignant à quel point celui-ci est lié aux visées émancipatrices de l'agence de l'eau du bassin de

\footnotetext{
${ }^{2}$ La loi sur l'eau de 1998 prévoit en effet que cette disposition soit déléguée aux Catchment Management Agencies (phase 3 du processus de délégation de prérogatives nationales aux agences de bassin dans le cadre de la décentralisation).
} 
l'Inkomati-Usuthu (IUCMA) par rapport au ministère de l'Eau (Department of Water Affairs - DWA). Enfin nous évoquons le cas particulier de l'opérationnalisation du dispositif dit du «débit environnemental» dans le cadre de ce modèle real-time, qui a été l'occasion pour l'IUCMA de redéfinir cette disposition et de l'artificialiser. Notre thèse est que l'utilisation du modèle real-time n'a pas seulement eu pour effet de neutraliser l'application d'un instrument d'action publique (Lascoumes et Le Galès, 2004), mais qu'elle redessine également les contours d'une des mesures-phares de la loi de réforme sur l'eau sud-africaine. En ce sens, parce qu'il redéfinit les objectifs de la loi, le modèle technique ne se contenterait pas de gérer, d'un point de vue purement technique, la ressource en eau, mais il se transformerait en un instrument de gouvernement de cette ressource. De plus, loin des difficiles négociations politiques auxquelles on aurait pu s'attendre, peu de discussions ont eu lieu en fait, ce qui illustre les effets d'autorité dont de tels dispositifs peuvent se prévaloir et mérite d'autant plus qu'on déconstruise ces dispositifs.

Notre démarche est fondée sur vingt-cinq entretiens menés entre 2013 et 2015 essentiellement avec les principaux acteurs de la gestion de l'eau dans le bassin de la Crocodile et au sein du DWA, ainsi que sur la lecture d'une abondante littérature grise émanant de l'IUCMA et du DWA sur les dispositifs de gestion de l'eau dans ce bassin versant ${ }^{3}$.

\section{S'adapter à la variabilité, limiter la pénurie?}

\section{La variabilité climatique «predictably unpredictable » sur la Crocodile}

Comme la plupart des rivières sud-africaines, la Crocodile est marquée par une forte variabilité climatique. Située dans la province du Mpumalanga, au nordest de l'Afrique du Sud, cette rivière prend sa source dans les montagnes du Steenkampsberg au nord de Dullstroom et rejoint à la frontière mozambicaine la Komati, qui se jette dans le nord de la baie de Maputo. $\mathrm{Si}$ son débit annuel moyen est relativement abondant (il est estimé à environ 1,13 kilomètres cubes par an, soit $\left.36 \mathrm{~m}^{3} \cdot \mathrm{s}^{-1}\right)$, il varie fortement entre la saison sèche et la saison humide de novembre à mars, mais également d'une année sur l'autre. L'alternance catastrophique de longues années sèches et d'inondations rend ce cours

\footnotetext{
${ }^{3}$ Cette recherche a fait l'objet de deux enquêtes exploratoires en mars 2011 (entretiens préliminaires pour le Mhlatuze) et décembre 2014 (enquête de terrain sur la Tosca-Molopo). Deux enquêtes de terrain ont été menées en avril 2013 sur la Komati et en mars 2015 sur la Crocodile dans le cadre de l'encadrement du mémoire de master de C. Chialva (2015).
}

d'eau, pour reprendre l'expression de l'hydrologue sudafricain B. Davies, naturellement "predictably unpredictable» (Fig. 1).

Cette variabilité climatique rend particulièrement difficile la gestion de ce cours d'eau, qui n'est régulé que par un seul barrage important, le Kwena Dam (réservoir de 167 millions de mètres cubes, soit $1 / 6$ du débit), érigé en 1984. Et ce d'autant plus que le Kwena Dam est situé très en amont, dans la partie la plus arrosée du bassin (environ $1200 \mathrm{~mm}$ de précipitation par an), à plus de 250 kilomètres des basses terres plus sèches $(600 \mathrm{~mm}$ de précipitation) qui en ont le plus besoin.

\section{Une rivière sur-utilisée?}

Si le bassin versant de la Crocodile couvre une superficie relativement modeste, à savoir $10450 \mathrm{~km}^{2}$, il comprend plusieurs zones économiques majeures, soit sur les 320 kilomètres de l'amont vers l'aval, le territoire dédié à l'agroforesterie $\left(1775 \mathrm{~km}^{2}\right.$, principalement en eucalyptus et pins), qui a un impact significatif sur la ressource puisqu'elle réduit d'environ $15 \%$ le débit en aval, puis l'aire d'alimentation de captage pour la ville de Nelspruit et enfin, avant la confluence de la rivière avec la Komati, la zone agricole du Lowveld, un haut lieu de l'agriculture irriguée, notamment de la culture de canne à sucre (12500 hectares), grande consommatrice en eau (Fig. 2). Le bassin de la Crocodile, et plus largement de l'Inkomati, se situe également dans un haut lieu du tourisme du pays, avec la présence du parc naturel national du Kruger situé en aval et pour lequel la Crocodile s'avère d'une importance écologique stratégique. En tant que rivière transfrontalière, elle doit également respecter un débit minimum de 2 mètres cube/seconde à la frontière en accord avec le Pigg's Peak Agreement de 1991. Cette demande en eau vient s'ajouter aux autres usages principalement agricoles déjà évoqués et fait planer la menace d'une "sur-allocation » de la ressource. En effet, d'après B. Jackson (2014, p. 39), qui est en charge des données statistiques de la IUCMA, la demande en eau sur le bassin de la Crocodile serait de 1000 millions de mètres cubes pour une ressource effectivement disponible de 870 millions de mètres cubes. La Crocodile ne parviendrait, ainsi, à couvrir que $87 \%$ des besoins actuels des usagers de l'eau, dont seulement $74 \%$ des besoins des irrigants et $95 \%$ des besoins domestiques (IUCMA, 2010).

Des études tendent également à montrer que la mise en œuvre du débit environnemental (appelé «ecological reserve» - réserve écologique - en Afrique du Sud) en application de la loi de réforme sur l'eau de $1998^{4}$ pourrait aggraver la situation de déficit hydrique

\footnotetext{
$\overline{{ }^{4} \text { National Water Act } n^{\circ}} 36$ of 1998.
} 
Débits annuels moyens de la Crocodile River à

Badfontein

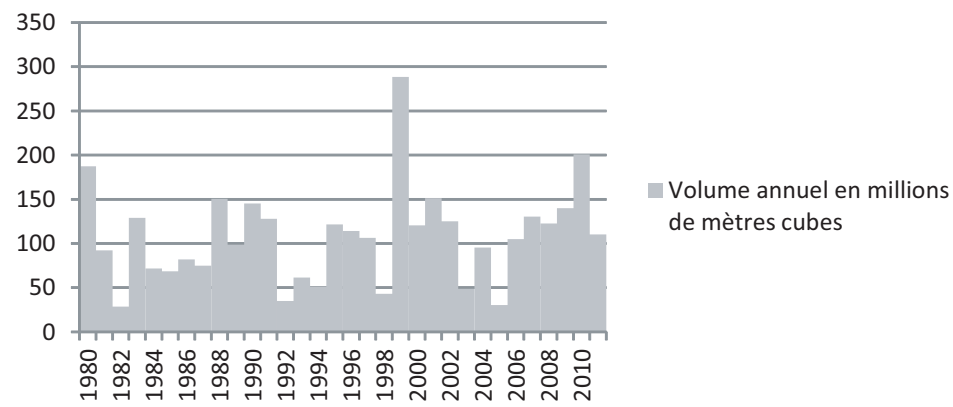

Source :http://riverops.inkomaticma.co.za/

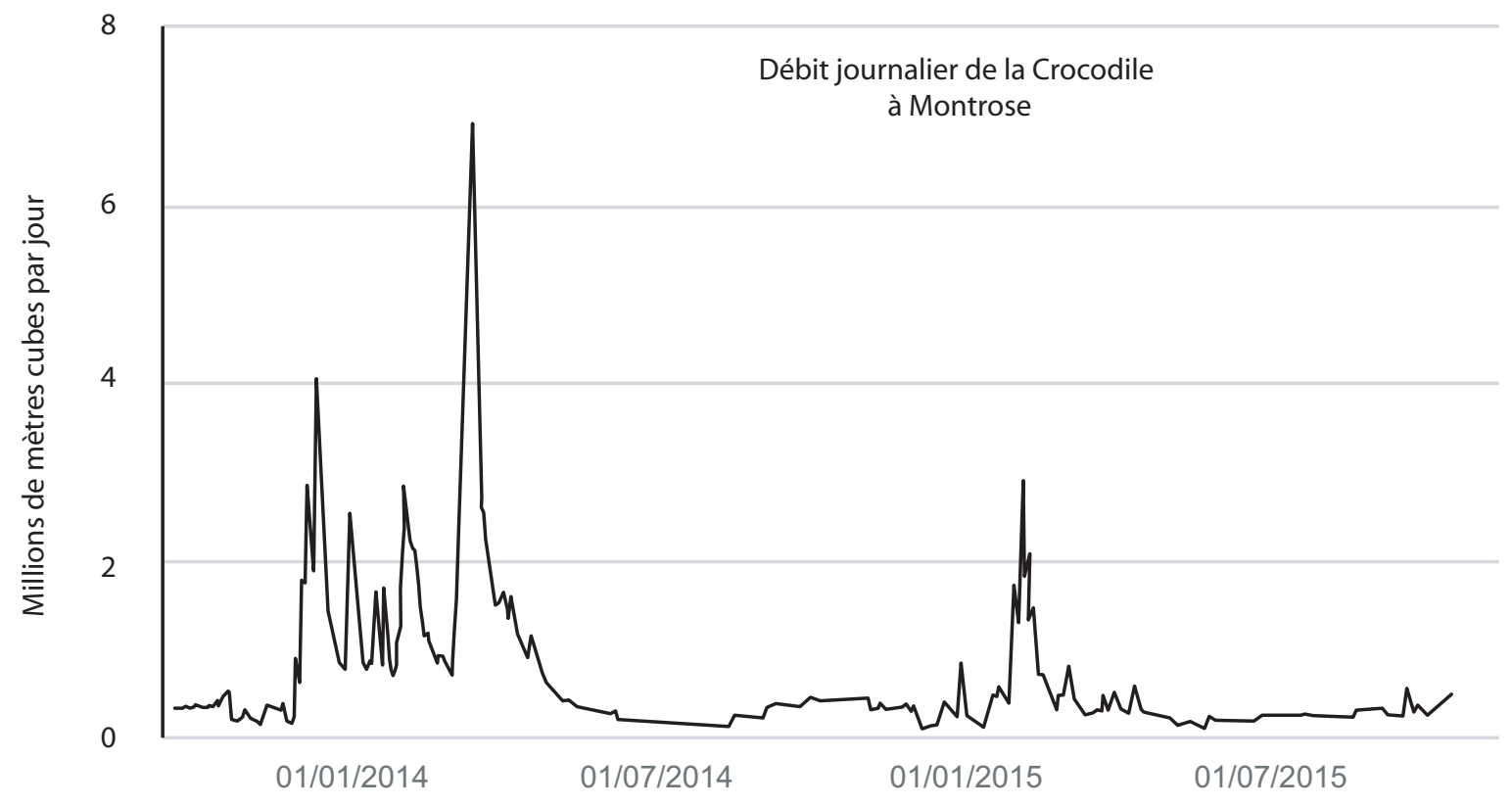

Fig. 1. Débits caractéristiques de la Crocodile River.

Cette figure montre deux échelles temporelles de la variabilité hydrologique de la Crocodile River. Le débit annuel à Badfontein, en aval du Kwena Dam montre l'alternance d'années humides et d'années sèches. On remarque notamment la succession d'années déficitaires au début des années 1980, 1990 et 2000, qui ont fortement marqué les esprits, alors que les années 2010 ont été pour l'heure relativement bien arrosées. Mais le graphique du débit journalier à Montrose, quelques kilomètres en aval, montre l'extrême variation des débits au cours d'une année : l'essentiel de l'écoulement a lieu de novembre à mars, avec des pics de crue très marqués, alors que pendant la plupart de l'année 2014, d'avril à décembre, le débit n'a été soutenu que par les lâchers d'eau du barrage de Kwena.

(Aurecon, 2012). Le débit environnemental correspond à un débit d'eau visant à couvrir a minima les besoins de l'écosystème aquatique. La réserve est le seul droit à l'eau reconnu par le législateur, les anciens droits ripariens ${ }^{5}$ ayant été abolis. Elle est prioritaire sur tous les autres

\footnotetext{
${ }^{5}$ Ces droits « ripariens » ou riparian rights en anglais datent du début de la colonisation. Ils donnent un droit sur l'eau à tout propriétaire riverain d'un cours d'eau. Autrefois très étendus, ils ont été limités par la loi sur l'eau de 1956, mais restaient considérés comme le fondement du droit prééminent des agriculteurs sur l'eau dans ce pays aride.
}

usages, ce qui pourrait potentiellement signifier moins d'eau pour les agriculteurs notamment, dont les usages ne sont plus prioritaires et doivent être désormais autorisés.

Enfin, il est question de revoir les obligations internationales et le débit minimum à la frontière mozambicaine ainsi que de permettre aux agriculteurs noirs émergents, dans un souci d'équité, de bénéficier d'un permis pour l'irrigation alors qu'ils en étaient très souvent privés jusque-là. La demande en eau pourrait donc être amenée à augmenter. Et lors des épisodes de sécheresse, des restrictions pourraient être alors appliquées aux agriculteurs. 


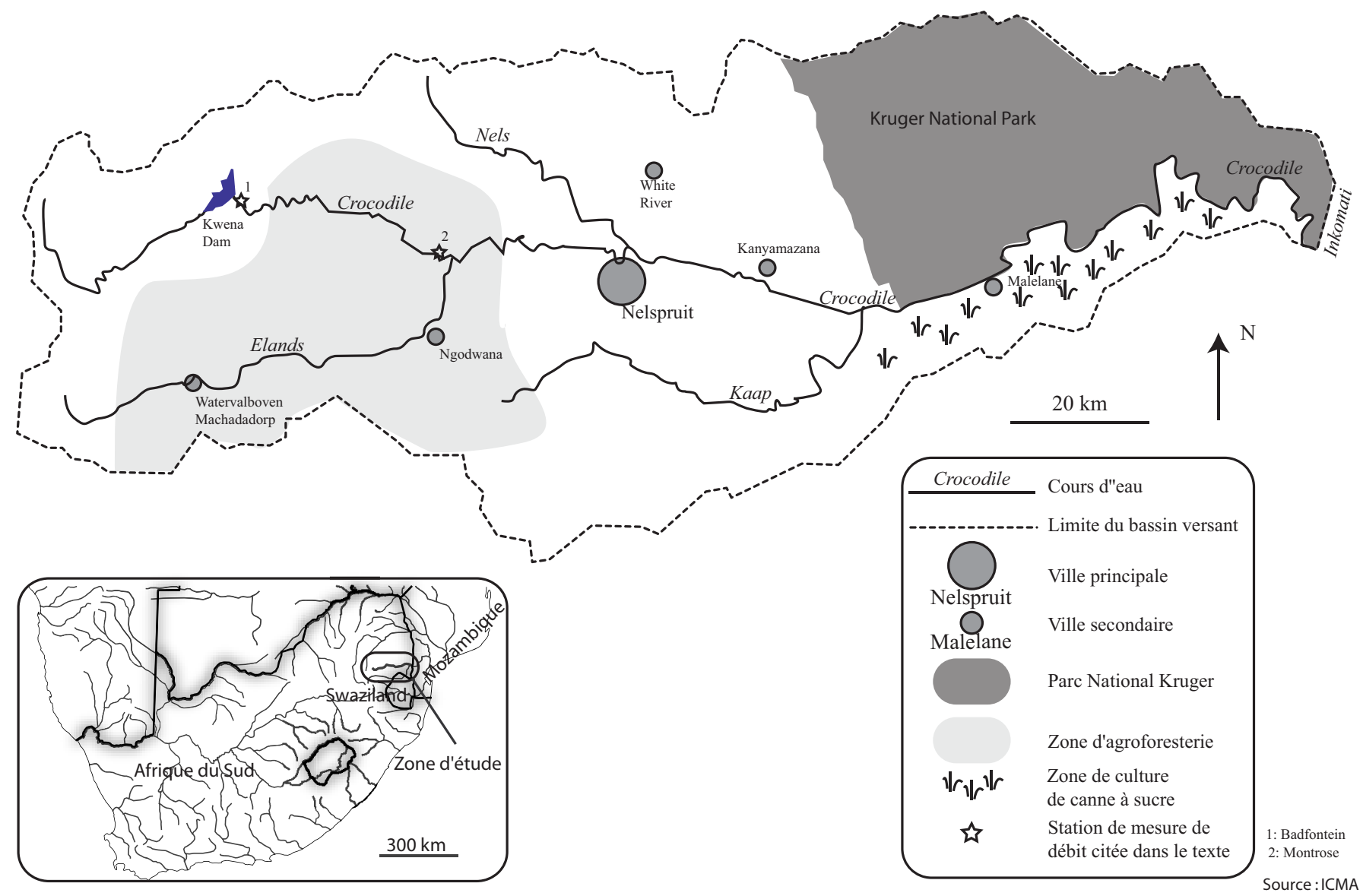

Fig. 2. Le bassin de la Crocodile.

\section{État des débats sur la production sociale de la pénurie}

Le cas de la Crocodile River en Afrique du Sud recoupe les débats actuels autour de l'analyse des discours sur la pénurie. Adoptant une approche s'inspirant des travaux de la Political Ecology, nous rejoignons les auteurs qui considèrent la pénurie comme un phénomène avant tout socialement construit et géographiquement et historiquement situé (Kaika, 2003 ; Noemdoe et al., 2006 ; Mehta, 2011 ; Buchs, 2016). K. Bakker (2000) définit ainsi la pénurie qui a touché cette région comme : «real, like nature, narrated, like discourse, and collective, like society». Tout comme dans le bassin de la Crocodile, cette pénurie est le fruit de «three interrelated practices: meteorological modeling, demand forecasting, and corporate restructuring and the regulatory "game" [...]. The drought was authorless, unintended, yet nonetheless had a kind of economic intelligibility; it was, in a limited sense, the least-cost option for Yorkshire Water Services given the constraints of the post-privatization regulatory game». De même, dans leur étude sur la pénurie d'eau dans les îles Canaries, Aguilera-Klink et al. (2000) soulignent que «the water scarcity in Tenerife is not physical or natural, but rather a socially constructed one, stemming from a set of social processes that reflect the conflicts concerning the desirable kind of society and social order $»$. Notre posture au sein de cet article est également de démontrer que la pénurie est plus la résultante du mode de gestion de la ressource qu'une réelle fatalité. Loin de constituer une fatalité, cette pénurie procède en fait d'un choix politique en faveur d'un statu quo qui bénéficie essentiellement aux irrigants blancs, dans un contexte marqué par une forte asymétrie dans l'accès à la ressource en eau. En effet, les travaux de Cullis et van Koppen (2007) montrent qu'en dépit des ambitions de redistribution des droits à l'eau affichés dans la loi de réforme sur l'eau de 1998 (NWA), plus de dix ans après la fin de l'apartheid, la population noire ne cumule toujours que $5 \%$ des usages en eau pour l'irrigation alors qu'elle représente $98,8 \%$ de la population rurale, tandis que les agriculteurs commerciaux blancs en consomment $95 \%$ tout en ne représentant que $1,2 \%$ de cette même population.

Pour analyser la «construction» de cette pénurie, Aguilera-Klink et al. (2000) proposent une méthode qui prend en compte dialectiquement les processus sociaux 
et environnementaux: "One could say that an understanding of social processes is needed for water (environmental) valuation and an understanding of the environment (water) is needed for social process valuation. » Les auteurs expliquent enfin comment les innovations techniques sont utilisées pour solutionner des problèmes qui sont avant tout sociaux, soit «[t]he search for technological "solutions" to the problems of social scarcity in order to avoid having to challenge water owners' vested interests $\gg$. C'est dans cette perspective que nous avons étudié le cas de la Crocodile, en nous attachant particulièrement à cerner les dispositifs techniques qui visait à «naturaliser» la question de la pénurie, effaçant les héritages de la période d'apartheid et à produire des méthodes de gestion de l'eau « indiscutables ».

\section{La mise en œuvre neutralisée du compulsory licensing}

\section{Un dispositif national complexe}

En dépit de l'abolition des anciens droits ripariens et de l'avènement depuis 1998 d'un régime d'autorisation préalable (licensing), le NWA n'a jamais voulu abroger purement et simplement les volumes d'eau obtenus sous l'ancien régime. Cela signifie que le DWA accorde, dans la plupart des cas, la possibilité d'obtenir un permis d'un volume équivalent aux anciens droits, pour peu que l'irrigant ait correctement enregistré ses usages sous l'ancien régime. Ce sont seulement dans les cas problématiques où la ressource se trouve en situation de stress hydrique que l'on a la possibilité d'avoir recours au compulsory licensing. Celui-ci autorise les autorités publiques à réduire les volumes des allocations déclarées. Cette conversion des droits ripariens en autorisation administrative s'effectue au travers de la détermination de ce que le législateur a appelé les «existing lawful uses». Cette conversion, qui n'a rien d'automatique, doit suivre une procédure complexe consistant à vérifier la réalité des usages de l'eau déclarés sous l'ancien régime (avant réforme) au travers des étapes dites de «validation» et de "vérification» de ces droits.

Pour le décrire succinctement, le processus de vérification concerne la légalité des prélèvements en eau : il est censé vérifier l'existence juridique d'une autorisation de prélèvement dans la ressource. Avant cela cependant, le processus de validation cherche à vérifier l'utilisation «effective» des volumes d'eau déclarés. En effet, une pratique courante sous l'ancien régime de droit riparien, quand le prix de l'eau était faible, consistait pour les irrigants à faire enregistrer, en prévision d'investissements futurs, des volumes qu'ils n'utilisaient pas encore. Pour le DWA, de telles pratiques ont permis dans des zones comme Mhlatuze de revenir à la normale sans avoir à recourir au mécanisme du compulsory licensing car la sur-allocation de la ressource n'existait que sur le papier, pas en réalité. Au moment de la vérification, les droits à l'eau déclarés mais non utilisés en pratique étaient donc supprimés selon le principe «use it or lose it». Une difficulté cependant pour les autorités étaient de connaître ces usages, la réalité de l'usage effectif de ces droits n'ayant jamais été historiquement attestée en l'absence de dispositif de mesure des prélèvements. En effet, avant 1998, les prélèvements d'eau ne faisaient pas encore l'objet d'un recensement comme cela doit être le cas aujourd'hui sous un régime d'autorisation. Certes, à l'époque, il convenait d'enregistrer les droits à l'eau pour lesquels les titulaires s'acquittaient d'une redevance plutôt modique. Cependant, cet enregistrement des droits se faisait au niveau local, le plus souvent au niveau des «irrigation boards » (syndicats d'irrigants) quand ceux-ci existaient. Ces données individuelles n'étaient donc pas centralisées au niveau du ministère. Dans un contexte post-apartheid où règne une forte défiance entre syndicats d'irrigants à majorité blanche et nouvelles élites noires du DWA, il était à prévoir que les syndicats d'irrigants seraient plus que réticents à partager ces précieuses informations avec le DWA en charge initialement du processus de compulsory licensing. Or, ces informations sont nécessaires pour mener à bien le processus ${ }^{6}$.

Les autorités ont donc été amenées à devoir reconstituer a posteriori ces usages, en prenant comme période éligible celle de 1996 (date du White Paper sur la réforme de l'eau) à 1998 (moment de l'entrée en vigueur du NWA). Pour ce faire, de nombreuses informations devaient être collectées. Pour déterminer les usages, il fallait connaître précisément à la fois les surfaces irriguées de l'époque et qui plus est, le type de cultures irriguées puisque les besoins en eau diffèrent d'une plante à une autre. Il a donc fallu recourir, entre autres, à la télédétection et aux images satellites (SPOT et Landsat) pour les années 1996 et 1998.

\section{La Crocodile: une région pilote?}

Mettre en œuvre la procédure de compulsory licensing n'était donc pas chose aisée en Afrique du Sud dans un contexte où les informations sur les usages de l'eau avant la loi de réforme sur l'eau de 1998 sont difficilement disponibles. Pour ce qui concerne plus

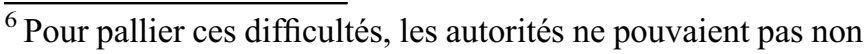
plus compter sur les mesures effectuées par les pompes des irrigants car toutes les pompes ne disposaient pas encore de compteurs. Les instruments de mesure pour les pompes des irrigants sont devenus obligatoires depuis 2014 seulement (Notice 1141 of 2014, JO du 11 décembre 2014).
} 
précisément le bassin de la Crocodile, il semble à l'heure actuelle que le recours au compulsory licensing apparaisse des plus hypothétiques. En effet, s'il y est fait référence dans certains documents officiels jusqu'à la fin des années $2000^{7}$, à partir de 2012, qui a vu la délégation à l'agence de l'eau d'un certain nombre de fonctions précédemment assurées par le ministère de l'Eau, il n'en est plus fait mention. À la différence des trois autres sites pilotes du compulsory licensing (Mhlatuze, Tosca Molopo et Jan Dissels), il est notable également que la procédure de compulsory licensing n'a jamais été publiée au journal officiel dans le cas du bassin de l'Inkomati-Crocodile. Le site internet du ministère de l'Eau n'indique plus non plus la Crocodile comme un site pilote $^{8}$. Pour autant, tout usage de cet outil n'a pas été officiellement écarté, notamment au niveau local. Pour l'ancien directeur du bureau régional du ministère de l'Eau, toujours en poste au DWA de la province du Mpumalanga, le bassin de l'Inkomati fait toujours partie d'une zone prioritaire pour le compulsory licensing 9 .

\section{Des résultats divergents selon les experts locaux et nationaux}

Eu égard à la complexité de la tâche, les services centraux du DWA vont recruter une équipe de consultants pour mener une étude sur le terrain dans le bassin de la Crocodile en 2008-2009. Movik (2012) évoque cependant les contradictions entre les premiers résultats auxquels aboutissent les consultants et les calculs préliminaires effectués par les services du DWA au niveau provincial. Ces derniers, considérant la Crocodile en état de sur-allocation de la ressource, refusaient d'octroyer aux agriculteurs noirs émergents de nouveaux permis d'eau. Pour l'équipe de consultants, il y avait au contraire plus d'eau disponible que le bureau régional du DWA ne voulait le concéder.

Finalement, achoppant sur trop de difficultés et révélant trop d'incohérences, le travail de ces consultants commissionnés par le DWA n'aboutira pas. L'étude Validation \& Verification sera confiée par la suite en 2010 à l'IUCMA qui va recruter ses propres consultants et reprendre entièrement le processus. Au vu des premières

\footnotetext{
${ }^{7}$ Develop a framework for water allocation to guide compulsory licensing in the Inkomati Water Management Area, DWAF \& Inkomati WMA, 2007; ou certains rapports de recherche encore en 201: MSIBI, M.I., DLAMINI, P.Z.; Water Allocation Reform in South Africa: History, Processes and Prospects for Future Implementation, Report to the Water Research Commission, 2011.

${ }^{8}$ http://www.dwaf.gov.za/ (consulté le 20/05/2015).

${ }^{9}$ Entretien avec le directeur du DWA Mpumalanga, Bronkhorstspruit, mars 2015.
}

conclusions de ce $\operatorname{travail}^{10}$, il semblerait qu'une opportunité de diminuer le niveau des allocations des irrigants ait été volontairement écartée par l'IUCMA ${ }^{11}$, en dépit des preuves manifestes de l'augmentation illégale des prélèvements par les agriculteurs commerciaux blancs entre 1998 et aujourd'hui ${ }^{12}$. Les gestionnaires de l'IUCMA ont préféré fermer les yeux sur ces pratiques en considérant qu'établir les preuves de cette augmentation illégale de la part des agriculteurs commerciaux relevait d'une procédure fastidieuse et à l'issue incertaine ${ }^{13}$.

Pour l'IUCMA, un processus de compulsory licensing ne s'impose pas non plus. D'abord parce qu'un des objectifs de ce dispositif réglementaire, qui est de permettre un accès à l'eau pour les agriculteurs noirs émergents, aurait été automatiquement atteint grâce à l'une des plus grandes opérations de redistribution foncière d'Afrique du Sud, qui s'est déroulée à Tenbosch sur le territoire de la Crocodile. Cette opération aurait eu pour effet de permettre d'atteindre automatiquement les objectifs de «transformation raciale» des usages de l'eau puisque près de $45 \%$ des anciens permis d'eau d'irrigation dans le secteur auraient été ainsi transférés aux agriculteurs noirs émergents de Tenbosch ${ }^{14}$. Cependant, en l'absence de soutien de l'État, ces agriculteurs émergents peinent à maintenir une activité agricole. Certains d'entre eux ont donc temporairement accepté de transférer leurs permis à d'autres membres, agriculteurs commerciaux blancs, du syndicat d'irrigants ${ }^{15}$. Ladite

\footnotetext{
${ }^{10}$ Qui va encore durer 18 mois (étape de la vérification) avec la confrontation des résultats de l'inventaire de l'IUCMA avec les dires des agriculteurs pour les cas où des disparités ont pu être relevées entre les déclarations initiales des agriculteurs et la vérification opérée par l'IUCMA.

${ }^{11}$ Comme pour Mlhatuze, sans même avoir besoin de recourir à une procédure formelle de compulsory licensing.

${ }^{12}$ Movik (2012, p. 129) évoque les allégations publiques d'un consultant du DFID avançant que depuis 1998 on pouvait repérer une expansion illégale dans le bassin de l'Inkomati de plus de 17000 ha irrigués.

${ }^{13}$ Entretien avec le manager de l'IUCMA, Nelspruit, mars 2015. De leur côté, les irrigants déclarent certes des surfaces irriguées plus grandes que celles utilisées, mais étant donné qu'ils utilisent des technologies d'irrigation plus économes en eau, in fine le niveau de leurs prélèvements n'aurait, selon eux, pas augmenté. Ces pratiques illégales pourraient également concerner aussi certains agroforestiers émergents.

${ }^{14}$ Entretien avec le manager de l'IUCMA, Nelspruit, mars 2015.

${ }^{15}$ Cette pratique est tolérée dans la mesure où elle est organisée au sein d'un même syndicat d'irrigants. Les autorités publiques sont par ailleurs plutôt réticentes vis-à-vis de l'échange marchand des anciens droits à l'eau (Movik, 2012, p. 42 ; interview de l'ingénieur en charge des systèmes d'irrigation, TSB company, Malelane, mars 2015).
} 
transformation des usages de l'eau demeure donc pour l'instant purement cosmétique. D'autant plus que ces transferts se feraient à un prix très bas, de l'ordre de $1000 \mathrm{ZAR} / \mathrm{ha}^{16}$. L'objectif de redistribution des droits à l'eau vers les populations noires historiquement défavorisées semble donc avoir été neutralisé alors même que l'accès à l'eau et le partage équitable de la ressource sont, depuis vingt ans, un enjeu majeur de la reconstruction d'une société démocratique au sein de l'Afrique du Sud post-apartheid ${ }^{17}$. Quant au compulsory licensing, il semble être durablement suspendu, surtout depuis que l'IUCMA a mis au point un modèle d'aide à la décision qui a pour ambition d'optimiser les décisions de lâchers de barrage et éviter au maximum les gaspillages d'eau. C'est dans ce contexte, et au-travers de ces dispositifs, que la situation de pénurie potentielle se retrouve requalifiée et redéfinie.

\section{Gouverner par les modèles : les enjeux de la mise en place d'un modèle real- time}

\section{Un modèle national et ses limites}

Comme nous l'avons vu, une des caractéristiques de la Crocodile est d'être marquée par une forte variabilité climatique naturelle. Ce trait commun à la plupart des hydrosystèmes sud-africains a fait l'objet de nombreuses recherches de la part de la communauté scientifique sudafricaine. Le modèle le plus abouti actuellement, le Water Resources Yield Model, dit modèle Yield ${ }^{18}$, permet d'allouer les ressources en eau dans un bassin versant sur une base annuelle en fonction de la disponibilité prévisible en eau du système. L'objectif en cas d'année sèche est d'assurer l'approvisionnement en eau prioritairement aux villes, aux industries stratégiques et aux États riverains, et donc de restreindre les irrigants en premier lieu. Ce modèle a été principalement conçu pour gérer le vaste système de transfert d'eau et de barrages sud-africain, et plus particulièrement pour assurer l'alimentation en eau de Johannesburg et Pretoria.

Au niveau du bassin de la Crocodile, ce modèle est moins adapté, car le cours d'eau n'est contrôlé que par un seul barrage, situé très en amont. De plus, il est également peu utile pour les principaux utilisateurs d'eau que sont les agriculteurs majoritairement blancs

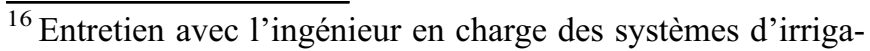
tion, TSB company, Malelane, mars 2015.

${ }^{17}$ À ce titre, l'adoption de la loi de réforme sur l'eau votée en 1998 fut un des projets prioritaires du nouveau régime politique de 1994.

${ }^{18} \mathrm{Ce}$ modèle fut mis au point dans les années 1980 (Basson et al., 1994).
}

qui se trouvent à l'extrémité aval du cours d'eau. En effet, en fonction de leur permis, les agriculteurs irrigants se voient attribuer un volume d'eau global correspondant à leur allocation pour l'année hydrologique. Les irrigants ont cependant tout intérêt à faire usage de cette allocation au moment opportun, pour suppléer l'absence de précipitations. Ces irrigants adressent donc des requêtes auprès du gestionnaire de la ressource, qui, en fonction de la disponibilité en eau dans le barrage, les autorisera à prélever dans la ressource une part de leur allocation annuelle. De là la forte dépendance des irrigants vis-à-vis des gestionnaires du barrage en cas de sécheresse.

De ce point de vue, le système a parfois été critiqué pour sa tendance à être assez rigide, planifiant sur des intervalles de temps trop longs les besoins en eau des irrigants. En effet, avec ce modèle Yield, les règles d'opérationnalisation du système sont décidées une fois par an seulement, et révisées tous les trimestres. Ainsi, ces agriculteurs étaient en quête d'une manière de gérer de façon plus réactive la ressource: une manière qui permette de s'adapter plus rapidement, c'est-à-dire au jour le jour plutôt qu'un an à l'avance, à l'évolution favorable ou défavorable des conditions pluviométriques.

\section{La création d'un modèle local comme mode d'affirmation institutionnel}

C'est ce que propose de faire un nouveau modèle dit en temps réel (real-time). Il émane d'une initiative des agriculteurs irrigants sur la rivière Komati, un autre affluent de l'Inkomati, pour contrer les décisions des gestionnaires de l'eau de la Komati Basin Water Authority (KOBWA $)^{19}$ jugées trop restrictives. Ces irrigants réclamaient en effet une gestion plus minutieuse de la ressource afin de limiter toute $\left\langle\right.$ perte ${ }^{20}$ et ainsi tenter de toujours faire plus avec moins d'eau disponible. Ils ont ainsi commandé une étude de faisabilité à un ingénieur-consultant ${ }^{21}$ qui par la suite va travailler en tandem avec l'IUCMA pour mettre au point un système similaire sur la Crocodile. Les premières réflexions

\footnotetext{
${ }^{19}$ La KOBWA gère les barrages de Nooitgedag et Vygeboom sur la Komati.

${ }^{20}$ Comme par exemple un lâcher de barrage prévu de longue date mais à une période non optimale compte tenu des informations actualisées sur l'arrivée de précipitations.

${ }^{21}$ Cet ingénieur-consultant local a initialement mis au point ce modèle real-time DSS avec l'aide de DHI, une firme internationale de consulting d'origine danoise (voir: DHI, 2011. Real-Time Management of a River highly in demand: Security and efficiency for the Crocodile River, across borders and competing interest, Johannesburg, South Africa).
} 
datent d'octobre 2009, au moment de l'arrivée du nouveau personnel à l'IUCMA nouvellement créée, dont une des attributions est d'être en mesure d'opérationnaliser un système de restrictions, parfois sévères, des allocations en eau sur les rivières dont elle a la responsabilité, dans l'éventualité d'épisodes de sécheresse.

Le modèle real-time est composé de deux logiciels, l'un relatif à la planification de long terme, l'autre pour les opérations au jour le jour. Le premier prend en compte les prédictions climatiques, les volumes d'eau dans le barrage et le débit de la rivière afin de générer des scénarii de disponibilité en eau et de restrictions potentielles. Le second a recours à un dispositif de surveillance actualisé des précipitations et des débits et de prévision de ceux à venir. Le modèle real-time requiert une masse de données considérable (sur les précipitations, les usages réels de l'eau, etc.), données qui n'étaient pas disponibles avant une période récente. Comment expliquer alors l'investissement de l'IUCMA sur ce dossier? Il peut certes s'expliquer par l'intérêt personnel qu'un membre en particulier de l'agence de l'eau lui accorde mais il convient surtout de le comprendre comme un moyen pour l'IUCMA de s'imposer dans un paysage institutionnel où l'agence de l'eau subit de nombreuses concurrences, en premier lieu, de la part du DWA. En effet, si l'IUCMA a hérité en 2012, dans le cadre des politiques de décentralisation de la gestion de la ressource, de prérogatives autrefois dévolues au ministère de l'Eau, le DWA a démontré par le passé toute sa défiance vis-à-vis de ce processus (Bourblanc et Blanchon, 2014). Cela contribue à brouiller la lecture du jeu complexe des acteurs du bassin, dont le fonctionnement n'est toujours pas stabilisé. On peut parler ensuite de la concurrence de la KOBWA qui gère une partie de la ressource en eau sur le territoire de l'Inkomati. L'enjeu était donc pour l'agence de l'eau de trouver des alliés, notamment en entretenant de bonnes relations avec les agriculteurs commerciaux qui, par ailleurs, contribuent largement à financer les agences de bassin comme l'IUCMA. L'IUCMA est également dans une position de relative dépendance vis-à-vis des puissants syndicats d'irrigants $^{22}$ qui disposent de données exclusives sur les usages de l'eau de leurs adhérents, qu'ils sont peu prompts à partager (notamment avec le DWA) mais dont l'IUCMA a besoin pour gérer au mieux les ressources du

\footnotetext{
${ }^{22}$ Le bassin de la Crocodile River est composé de cinq Irrigation Boards : le Crocodile River Major Irrigation Board, le White River Valley Conservation Board, le Kaap Major Irrigation, le Sand River Irrigation Board et le Lows Creek Irrigation Board, ainsi que deux Water Users Associations théoriques (mais très peu fonctionnelles), l'Elands River et l'Upper Kwena.
}

bassin. Certaines concessions leur sont donc faites officieusement, comme par exemple, en cas de doute sur l'existence d'une augmentation frauduleuse des prélèvements autorisés, celui-ci bénéficie en général aux irrigants ; ou encore des concessions quant à la possibilité qui leur est accordée d'utiliser les «surplus water $»^{23}$, alors que réglementairement les agriculteurs ne peuvent pomper que dans la limite des volumes d'eau autorisés individuellement ${ }^{24}$. Par ailleurs, la portée «politique» $\mathrm{du}$ modèle real-time s'affirme également dans son utilisation pour calculer le débit environnemental prévu dans le National Water Act de 1998. En effet, le débit environnemental initialement déterminé par le DWA au niveau national va devoir être adapté avec la mise en place d'un système real-time. Une courbe indiquant des valeurs mensuelles, comme c'était le cas jusqu'à présent, ne suffit plus. Il convient d'affiner la mesure et de déterminer des valeurs au moins hebdomadaires. La collaboration entre l'IUCMA et l'ingénieur-consultant cité plus haut va représenter le travail le plus abouti en la matière.

\section{Mise en œuvre du débit environnemental et redéfinition des objectifs légaux}

Un débit environnemental préliminaire avait été déterminé temporairement par les écologues du DWA vers la seconde moitié des années $2000^{25}$. Néanmoins, il n'était pas encore appliqué, comme du reste dans la quasi-totalité des cas dans le pays (Bourblanc, 2013). La responsabilité du suivi de cette mise en œuvre revient désormais à l'IUCMA en vertu de la délégation de pouvoirs reçue en décembre 2010. Les autorités du parc naturel du Kruger situé en aval sont chargées, quant à elles, du dispositif de bio-surveillance sur le territoire du parc naturel ${ }^{26}$. Constatant le non-respect du débit environnemental, les autorités du parc naturel du Kruger avaient pris très tôt les devants en la matière. En effet, confrontées à des épisodes où les rivières du parc se retrouvaient totalement à sec, elles avaient pris, dès 2004, l'initiative de la création d'un forum, le Crocodile Catchment Forum, conçu comme une plateforme de dialogue entre représentants de divers groupes d'intérêt,

${ }^{23}$ Le «surplus water» correspond à l'excédent d'eau qu'un barrage laisse s'échapper par un déversoir en période de crue, quand le barrage est rempli.

${ }^{24}$ Entretien avec le manager de l'IUCMA, Nelspruit, mars 2015.

${ }^{25}$ Project WP 9133: Comprehensive Reserve Determination Study for Selected Water Resources (Rivers, Groundwater and Wetlands) in the Inkomati Water management Area, Mpumalanga.

${ }^{26}$ Section 18, National Water Act 36 of 1998. 
en premier lieu entre irrigants et groupes d'intérêt conservationnistes ${ }^{27}$. Grâce à l'existence de ce forum, les agriculteurs irrigants seraient passés d'une attitude de refus total de dialoguer avec les autorités du Kruger à une plus grande ouverture envers la réserve écologique ${ }^{28}$.

Quoi qu'il en soit, la mise en œuvre du débit environnemental représente un défi majeur dans un contexte marqué par la présence de barrages au fil de la ressource, barrages qui ont pour effet de modifier irréversiblement l'écoulement naturel de la rivière. Un des enjeux est donc de rendre compatible la mise en place de cet objectif avec les règles opérationnelles de gestion de la ressource en eau à partir du barrage. Avant même de procéder à sa mise en œuvre, néanmoins, les gestionnaires de l'IUCMA vont re-calculer le débit environnemental dans le cadre de la mise en place du modèle realtime, en dehors de la procédure réglementaire.

\section{Différentes approches pour déterminer le débit environnemental}

Les écologues du DWA étaient à l'origine de la détermination d'une réserve préliminaire, établie en 2008 (Louw et Huggins, 2008). Ces écologues recommandaient alors une réserve dite de classe $\mathrm{C}$, c'est-à-dire correspondant à une ressource modérément modifiée. Elle devait être mise en œuvre selon un principe de "gentleman agreement» en attendant la fin $\mathrm{du}$ processus, long et complexe, de détermination du débit environnemental en Afrique du $\mathrm{Sud}^{29}$. En effet, à la différence de la directive-cadre européenne sur l'eau par exemple, la législation sud-africaine en matière de débit environnemental n'impose pas de revenir à un «bon état écologique» sur tous les cours d'eau ${ }^{30}$. Ainsi, en fonction de l'écart entre l'état actuel de la ressource et l'état naturel, de la sensibilité écologique du milieu, de l'impact sur la ressource des différentes activités économiques, de l'importance que l'on accorde à la ressource ou encore de ce qu'il est

\footnotetext{
${ }^{27}$ Ce Crocodile Catchment Forum fut, par la suite, officiellement établi en 2010 en réponse à l'obligation législative d'établir des comités au sein des bassins versants.

${ }^{28}$ Entretien avec le directeur par intérim de l'IUCMA, Nelspruit, mars 2015.

${ }^{29}$ Pour la Crocodile, ce processus a franchi une étape décisive le 22 janvier 2016 avec la publication au journal officiel par la Ministre de l'Eau d'une proposition de classe de gestion (No 39614). Une consultation publique a été ouverte pendant 60 jours afin de recueillir tout commentaire sur cette proposition.

${ }^{30}$ Il existe 6 classes, de A, correspondant à l'état naturel, à F, correspondant à un état extrêmement modifié. Il n'est pas permis cependant de viser une classe de gestion de la ressource en-dessous d'une classe D (ressource largement modifiée).
}

réaliste d'atteindre en fonction des efforts que les différentes parties intéressées sont prêtes à consentir, une classe de gestion de la réserve doit être déterminée. Cette classe de gestion doit être déterminée par consensus entre le DWA et les différents groupes d'intérêt socioéconomiques au niveau de chaque cours d'eau.

Cette classe de gestion n'était pas encore déterminée quand les premières réunions du Crocodile Catchment River Operations Committee (CROCOC) sur la réserve ont débuté. Le CROCOC est un comité technique consultatif créé par l'IUCMA, rassemblant principalement des irrigants. Il a été mis en place pour mettre en œuvre le modèle real-time et a pour but d'assister un processus de prise de décision sur la base d'un consensus pour les opérations réalisées sur la rivière Crocodile. Il a un rôle de coordination de l'information et des opérations et il conseille les autorités en charge d'implémenter le système à partir du barrage Kwena. Or, le CROCOC avait calculé que la mise en œuvre de la réserve préliminaire déboucherait sur la réduction d'environ $30 \%$ des usages courants de la ressource sur la Crocodile $^{31}$. Les discussions sur le débit environnemental au sein du CROCOC ont duré près d'un an et demi avant qu'un accord puisse être trouvé sur la manière de procéder: "Ce qui s'est passé dans les discussions [au sein du CROCOC], c'est que les écologues [du DWA] demandaient beaucoup d'eau [pour l'écosystème] pour maintenir une réserve écologique de classe $\mathrm{C}$. Mais si vous regardez [...] les statistiques sur les débits actuellement observés [present day reserve], c'est bien plus bas que ce qu'une classe $\mathrm{C}$ requiert $^{32}$.»

Constatant cette tendance à la dégradation de l'état de la ressource, le CROCOC vise donc désormais le maintien du niveau actuel de la réserve (present day reserve) correspondant à une classe $\mathrm{D}$ plutôt qu'à la classe $\mathrm{C}$ recommandée pour la réserve préliminaire. En révisant à la baisse le niveau du débit environnemental, on découvre sans surprise, du coup, un taux de conformation au débit environnemental real-time aujourd'hui bien supérieur comparé aux périodes précédentes. Surtout, il est possible de repérer des différences majeures dans la conception de la réserve et la manière de la déterminer, selon que l'on possède plutôt un profil d'écologue ou un profil d'ingénieur de la ressource en eau. Ainsi l'ingénieur-consultant à qui l'on a confié la tâche de traduire la réserve pour la rendre compatible avec le modèle real-time va entreprendre cette tâche avec les compétences qui sont les siennes, à savoir celles non pas d'un hydrologue

\footnotetext{
${ }^{31}$ ICMA, Minutes of the $1^{\text {st }}$ CROCOC meeting, $6{ }^{\text {th }}$ Oct 2009.

${ }^{32}$ Entretien avec le Manager de l'IUCMA, Nelspruit, mars 2015.
} 
ou d'un écologue ${ }^{33}$, mais d'un ingénieur de l'eau. Sa méthode consistait à, d'un côté, additionner les usages connus de l'eau, et de l'autre, à utiliser un modèle hydrologique (modèle pluie-débit). Le débit environnemental est obtenu par la soustraction des premiers au second. Autrement dit, selon cette méthode, la réserve correspond au débit restant. L'ingénieur-consultant concède que sa méthode diffère de celle des scientifiques recrutés par le DWA pour déterminer le débit environnemental. Il revendique néanmoins : « J'ai testé mon modèle en le comparant avec la réserve déterminée par le DWA. J'ai pris cinq années de données et je suis parvenu à des résultats similaires au DWA [pour la réserve] ${ }^{34}$.»

De plus, la méthode est tributaire de l'existence de systèmes modernes de mesure des prélèvements. L'ingénieur-consultant reconnaît, ainsi, les limites de ce modèle qui ne permet pas de tenir compte des usages illégaux, pourtant très répandus : «Par contre, ma méthode ne marche pas ailleurs dans les rivières où il $\mathrm{y}$ a beaucoup d'inconnus, où les pompages ne sont pas tous mesurés, où il $\mathrm{y}$ a beaucoup d'usages non autorisés. J'ai essayé et la comparaison révélait de trop grandes différences ${ }^{35}$.»

Officiellement, le modèle utilisé par le CROCOC n'avait vocation qu'à mettre en œuvre la réserve écologique, pas à la re-calculer. In fine pourtant, le débit environnemental va être non seulement re-calculé lors de l'étape de la mise en œuvre mais l'on peut dire aussi qu'il va être redéfini. Il ne correspond plus au débit nécessaire à l'écosystème pour assurer sa durabilité. Il correspond plutôt désormais au débit restant dans la rivière une fois que lui ont été soustraits tous les autres besoins en eau qui, de fait, ont été servis prioritairement alors qu'en application de la loi, la réserve était censée avoir la priorité sur ces usages.

\section{Apprendre chemin faisant : la philosophie du «Strategic adaptive management » appliquée à la détermination du débit environnemental}

La méthode de détermination de la réserve désormais préconisée a donc fortement évolué. Si la réserve préliminaire calculée par les écologues du DWA était basée sur la méthode dite de l'instream flow requirement, il s'agit désormais de se focaliser sur un apprentissage chemin faisant, qui prête attention aux boucles de rétroaction et aux réactions du milieu. La proximité intellectuelle avec certains écologues du parc Kruger

\footnotetext{
${ }^{33}$ Profil que l'on retrouve majoritairement parmi les consultants ayant collaboré avec le DWA pour déterminer les débits environnementaux dans la plupart des rivières du pays.

${ }^{34}$ Entretien avec l'ingénieur-consultant ayant mis au point le modèle real-time, Nelspruit, mars 2015.

${ }^{35}$ Entretien avec l'ingénieur-consultant ayant mis au point le modèle real-time, Nelspruit, mars 2015.
}

avec lesquels l'ingénieur-consultant de l'IUCMA a pu collaborer (McLoughlin et al., 2011 ; Pollard et al., 2011) a contribué à la promotion de cette approche dite du strategic adaptive management. Comme le confie l'actuel directeur de l'IUCMA, ancien manager en chef du programme de conservation de la biodiversité aquatique du Kruger : «On observe le système chemin faisant, si on applique une réserve écologique d'un certain niveau, on vérifie que les poissons ne sont pas en train de mourir. Il s'agit de mettre en œuvre progressivement le débit environnemental ${ }^{36}$.»

Cela supposait de mettre en place un système de biomonitoring comme l'a fait le parc national du Kruger afin de surveiller l'état du milieu et vérifier que l'état de la rivière n'est pas en train de se détériorer ${ }^{37}$.

\section{Une réserve écologique artificielle? La saisonnalité à contre-courant du débit environnemental}

Une des caractéristiques de la région du Lowveld marquée par un climat semi-aride est le fait que, naturellement, la rivière cesse de s'écouler à certaines périodes de l'année. C'est justement dans ce contexte que les barrages ont été érigés pour compenser l'arrêt (naturel) de l'écoulement en période sèche. Or, le débit environnement tel qu'il est mis en œuvre par l'IUCMA n'en tient pas compte. Comme l'avoue un des écologues du parc Kruger qui s'exprime sur cette méthode du recours à des lâchers de barrage pour mettre en œuvre le débit environnemental: «On peut mettre en œuvre la réserve à travers des lâchers de barrage mais les lâchers sont programmés à la mauvaise période de l'année, d'août à octobre [...], c'est ce qu'on appelle une saisonnalité inversée $[. .$.$] , on augmente le débit de la rivière à une$ période où naturellement il est censé diminuer. Le gestionnaire du barrage effectue ces lâchers pour pouvoir fournir en eau les irrigants qui en ont le plus besoin à cette période $^{38}$.» Ceci fait écho aux travaux de van Vuuren (2012, p. 164) sur la rivière Pongola, un des hauts lieux de l'émergence du concept de réserve écologique en Afrique du Sud (Bourblanc, 2013). L'auteure souligne également l'ambiguïté de la mise en œuvre du débit environnemental sur ce terrain, avec des lâchers de barrages correspondant essentiellement aux besoins des producteurs de maïs irrigué mais à des périodes contreproductives pour la pérennité de l'écosystème, avec pour

\footnotetext{
$\overline{{ }^{36} \text { Extrait d'un entretien }}$ avec le directeur par intérim de l'IUCMA, Nelspruit, mars 2015.

${ }^{37}$ Entretien avec l'ingénieur-consultant ayant mis au point le modèle real-time, Nelspruit, mars 2015.

${ }^{38}$ Entretien avec le nouveau manager en chef du programme de conservation de la biodiversité aquatique du Kruger, Nelspruit, mars 2015.
} 
conséquence des stocks de poissons qui vont décliner rapidement entre 1984 et 2005 . Cette artificialisation du concept de réserve écologique n'est pas l'unique dilemme auquel doivent faire face les autorités du parc Kruger, qui pourraient être amenées à soutenir le projet d'un barrage pour assurer un débit écologique sur la Crocodile.

\section{Construire un nouveau barrage pour assurer le débit environnemental?}

Optant pour une approche pragmatique, les scientifiques du Kruger ont tablé, depuis le départ, sur un processus d'apprentissage au travers du Crocodile Catchment Forum pour accoutumer les irrigants notamment au principe du respect de la réserve écologique. Pour eux, l'enjeu n'est pas encore de déterminer très précisément une quantité d'eau satisfaisante pour assurer les fonctions vitales de l'écosystème, mais juste de faire en sorte que le principe d'un débit environnemental soit respecté. Cette attitude pragmatique peut s'observer à différents niveaux: «On doit défendre la biodiversité. Techniquement, vu nos mandats [faire respecter le National Environmental Management Act, le National Water Act, etc.], nous ne sommes pas censés approuver une catégorie $\mathrm{C}$, nous devrions recommander une classe A pour viser un écosystème presque «naturel», mais ça ne serait pas réaliste ${ }^{39}$.»

Le changement de position sur le barrage De Hoop récemment inauguré sur la rivière Olifants, au nord du parc, témoigne aussi de ce pragmatisme. En dépit de l'impact d'un barrage sur l'écologie du milieu, l'ancien manager en chef du programme de conservation de la biodiversité aquatique du Kruger confie: «J'ai réalisé une étude pour le Kruger, ses résultats montrent que les barrages sont un mal nécessaire [necessary evil], à condition qu'ils ne soient pas situé près du Kruger. Sans le barrage Kwena, les rivières dans le Kruger seraient à sec. On ne peut pas compter sur les précipitations uniquement. On est tributaire des lâchers de barrage ${ }^{40}$.»

Pour cette raison, les écologues du Kruger sont prêts à soutenir le projet de barrage sur la rivière Kaap, afin d'assurer le débit environnemental dans le bassin de la Crocodile, en dépit du paradoxe que nous soulignions précédemment $^{41}$. Sans ce nouveau barrage, en effet,

\footnotetext{
${ }^{39}$ Entretien avec le nouveau manager en chef du programme de conservation de la biodiversité aquatique du Kruger, Nelspruit, mars 2015. Le terme employé et traduit ici par «naturel» est «pristine», soit «immaculé, sans intervention humaine ».

${ }^{40}$ Entretien avec le directeur par intérim de l'IUCMA, Nelspruit, mars 2015.

${ }^{41}$ Entretien avec le nouveau manager en chef du programme de conservation de la biodiversité aquatique du Kruger, Nelspruit, mars 2015.
}

l'ingénieur-consultant de l'IUCMA prédit une détérioration certaine de l'état écologique de la rivière. De plus, la construction de ce nouveau barrage pourrait être entièrement à la charge des autorités du Kruger, les irrigants refusant à l'heure actuelle d'en supporter le coût ${ }^{42}$.

En résumé, l'exemple de la gestion de la réserve écologique sur la Crocodile est caractéristique du détournement au niveau local des politiques conçues au niveau national. L' « ecological reserve» a été en effet pensée pour préserver les écosystèmes dans une perspective de durabilité (le «For ever» du slogan du ministère de l'Eau). Elle devrait donc prévoir, pour ces hydrosystèmes marqués par la variabilité, des périodes d'assèchement complet, ainsi que des fortes crues. Mais elle a été mise en place dans des hydrosystèmes déjà fortement anthropisés, dans une perspective complètement opposée, puisqu'il s'agit de maintenir un débit, qui plus est en construisant des ouvrages visant à régulariser encore plus le débit du fleuve. En ce sens, la réserve écologique se rapproche de la définition des débits d'objectifs d'étiage en France analysés dans les travaux de S. Fernandez (2014). Le compromis «politique» sur la Crocodile River vise ainsi à limiter la variabilité naturelle du cours d'eau et à artificialiser une mesure censée protéger les écosystèmes naturels. Enfin, on peut émettre ici l'hypothèse que c'est le faible nombre de personnes capables de maitriser effectivement le modèle real-time (moins d'une dizaine en tout) qui permet ces détournements.

\section{Conclusion}

Nous avons étudié dans cet article trois dispositifs sociotechniques mis en œuvre sur la rivière Crocodile en Afrique du Sud: les processus de compulsory licensing, de real-time model et de détermination du débit environnemental. Cette analyse a été l'occasion de mettre en évidence à quel point ces dispositifs sociotechniques, loin de se contenter de gérer, d'un point de vue purement technique, la ressource en eau, se sont transformés en fait en instruments de gouvernement de cette ressource. En premier lieu, nous avons démontré à quel point la pénurie avec laquelle les gestionnaires de l'agence de l'eau doivent composer n'avait rien d'une fatalité et était pour une large part alimentée par leurs choix gestionnaires. L'attitude permissive envers les irrigants commerciaux blancs, alors qu'il existe de fortes suspicions d'usages illégaux, illustre ces choix

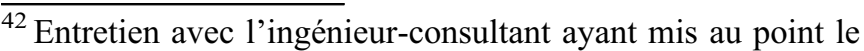
modèle real-time, Nelspruit, Mars 2015. Et ce en dépit du fait que les irrigants ont été ceux qui en premier ont réclamé - en vain, pendant des années - auprès du DWA la construction d'un tel ouvrage dans un contexte de pression accrue sur la ressource.
} 
qui renforcent une situation potentielle de pénurie. L'agence de l'eau a pu de plus se distinguer en neutralisant certaines dispositions réglementaires telles que le compulsory licensing par exemple. Certains dispositifs vont plus loin et renégocient les objectifs de l'action publique, sans toutefois engager de discussion politique ouverte et transparente sur le sujet. Il en est ainsi, par exemple, du débit environnemental, qui sera opérationnalisé par les gestionnaires du barrage Kwena qui vont en déterminer en particulier les composantes hebdomadaires, voire journalières. La réserve écologique est ainsi aujourd'hui réduite à la portion congrue et de fait redéfinie. Elle s'apparente beaucoup plus à l'approche française des débits d'objectifs d'étiage (Fernandez, 2014).

Certains de ces dispositifs dissimulent ainsi derrière leur complexité technique de nouvelles priorités : dans le contexte sud-africain, cela a pour conséquence de défendre le statu quo et donc de redéfinir l'agenda de transformation du pays. On peut même avancer le fait qu'en Afrique du Sud, les modèles (comme le modèle Yield dès les années 1980) ont toujours été un outil de préservation du pouvoir des ingénieurs du Department of Water Affairs face aux responsables politiques élus. Même une volonté politique forte comme celle de K. Asmal, ministre de 1994 à 1999, n'a jamais réussi à infléchir le rapport de force, et ses critiques sévères envers les grands barrages, au sein de la World Commission on Dams, n'ont pas empêché la construction de nouveaux ouvrages dans son propre pays.

Ainsi, l'étroitesse du monde de l'expertise sur l'eau en Afrique du Sud et sa concentration en quelques personnes ayant la même formation d'ingénieur civil en fait un cas particulier. Mais tout comme l'ont montré Fernandez et Debril (2016) à propos du débit d'étiage en France, les dispositifs sociotechniques que nous avons étudiés ici sont loin de se situer en aval du processus de décision. Ils ne découlent pas de choix préalablement établis dans la gestion de l'eau mais, bien plutôt, participent du cadrage des modalités de cette gestion. Autrement dit, ces dispositifs sociotechniques configurent, a priori, une manière particulière de gérer la ressource en eau et pré-emptent certains choix de gestion de cette ressource (qui sont essentiellement des choix politiques).

\section{Références}

Aguilera-Klink F., Perez-Moriana E., Sanchez-Garcia J., 2000. The social construction of scarcity. The case of water in Tenerife (Canary Islands), Ecological Economics, 34, 2, 233-245.

Aurecon, 2012. PRIMA (Progressive Realisation of the IncoMaputo Agreement). Close out report prepared for
Tripartite Technical Committee (TPTC) between Mozambique, South Africa and Swaziland. Report, Pretoria.

Bakker K.J., 2000. Privatizing water, producing scarcity: The Yorkshire drought of 1995, Economic Geography, 76, 1, 4-27.

Basson M.S., Allen R.B., Pegram G.G.S., van Rooyen J.A., 1994. Probabilistic Management of Water Resource and Hydropower Systems, Colorado, Water Resources Publications.

Bourblanc M., 2013. Les trajectoires bifurquées de la « Réserve écologique » sud-africaine: d'une logique aménagiste à une logique écologique, Autrepart, 2, 65, 27-45.

Bourblanc M., Blanchon D., 2014. The challenges of rescaling South African water resources management: Catchment management agencies and interbasin transfers, Journal of Hydrology, 591 (c), 2381-2391.

Buchs A., 2016. La pénurie en eau est-elle inéluctable? Bruxelles, Peter Lang.

Chialva C., 2015. La gestion d'une rivière dans un contexte de variabilité et d'incertitude. Mémoire de Master 2, NanterreLa Défense, Université de Paris Ouest.

Cullis J., van Koppen B., 2007. Applying the Gini coefficient to measure inequality of water use in the Olifants River water management area, South Africa. Research Report 113, Colombo, International Water Management Institute Research.

Davies BR, O’Keeffe J.H., Snaddon C.D., 1995. River and stream ecosystems in Southern Africa: Predictably unpredictable, in Cushing C.E., Cummings K.W., Minshall G.W. (Eds.), River and Stream Ecosystems, New York, Elsevier Press, 537-599.

Fernandez S., 2014. Much ado about minimum flows. Unpacking indicators to reveal water politics, Geoforum, 57, 258-271.

Fernandez S., Debril T., 2016. Qualifier le manque d'eau et gouverner les conflits d'usage : le cas des débits d'objectifs d'étiage (DOE) en Adour-Garonne, Développement durable et territoires, 7, 3 .

IUCMA, 2010. Inkomati Catchment Management Strategy, Nelspruit, IUCMA.

Jackson B., 2014. An adaptive operational water resources management framework for the Crocodile River catchment, South Africa. Mémoire de Master, Pietermaritzburg, Université du KwaZulu-Natal.

Kaika M., 2003. Constructing scarcity and sensationalising water politics: 170 days that shook Athens, Antipode, 35, 5, 919-954.

Lascoumes P., Le Galès P. (Dir.), 2004. Gouverner par les instruments, Paris, Presses de Sciences Po.

Louw M.D., Huggins G., 2008. Comprehensive reserve determination study for selected water resources (rivers, groundwater and wetlands) in the Inkomati water management area, Mpumalanga, Sabie and Crocodile systems: Desktop ecoclassification report. Report No. 26/8/ 3/10/12/002, Pretoria, Department of Water Affairs and Forestry (South Africa), Resource Directed Measures.

McLoughlin C., Mckenzie J., Rountree M., Grant R., 2011. Implementation of strategic adaptive management for 
freshwater protection under the South African national water policy. Research Report K5-1797/1/11, Pretoria, South-African Water Research Commission.

Mehta L. (Ed.), 2011. The limits to scarcity. Contesting the politics of allocation Abingdon, Routledge.

Movik S., 2012. Fluid rights. Water allocation reform in South Africa,Cape Town HSRC Press.

Noemdoe S., Jonker L., Swatuk L., 2006. Perceptions of water scarcity: the case of Genadendal and Outstations, Physics and Chemistry of the Earth, 31, 15-16, 771-778.

Pollard S., Mallory S.J.L., Ridell E., Sawunyama T., 2011. Towards improving the assessment and implementation of the Reserve: Real-time assessment and implementation of the ecological reserve. Final Report K8/881/2, Pretoria, Water Research Commission.

Van Vuuren L., 2012. In the footsteps of giants. Exploring the history of South Africa's large dams, Pretoria, Water Research Commission.

Zittoun P., 2013. Dispositif, in Casillo I. avec Barbier R., Blondiaux L., Chateauraynaud F., Fourniau J.-M., Lefebvre R., Neveu C., Salles D. (Dir.), Dictionnaire critique et interdisciplinaire de la participation, Paris, GIS Démocratie et Participation, http://www.participation-et-democra tie.fr/fr/dico/dispositif.

Citation de l'article : Bourblanc M., Blanchon D., 2017. Gérer ou gouverner la ressource en eau ? Modélisation en temps réel et gestion de la pénurie au sein de la rivière Crocodile (Afrique du Sud). Nat. Sci. Soc. 25, 2, 134-147. 